Vladimir Karpichev - Konstantin Sergeev - Aleksandra Bolotina

\title{
MODELING OF TECHNOLOGICAL PROCESSES OF MACHINE-BUILDING AND REPAIR MANUFACTURE
}

The proposed methods of constructing models of technological processes (TP) of production, repair and technical maintenance of objects in machine buildings, such as railway rolling stock, serves as the basis for development of information, mathematical and methodological support of the CAD system TP.

Applying of information technology to solve the problems of technological design has recently received intensive development. The research results show the effectiveness of the optimization approach to solving technological problems. Automation of the design of technological processes, based on use of digital technologies allows reducing the time for introducing new technical means and technologies into production, increasing the efficiency of production, repair and maintenance of objects in machine buildings.

Keywords: objects in machine buildings, railway rolling stock, production, repair and technical maintenance, technical designing, optimization methods, mathematical model of the technological process, digital technology, computer-aided design

\section{Introduction}

The systematic approach is the most general approach to designing complex objects. For a specialist in the field of systems engineering, they are obvious and natural, but their compliance and implementation are often associated with certain difficulties, due to the designing features. Mechanical engineers and repair enterprises use a systematic approach without recourse to system analysis manuals, but an intuitive approach without applying the rules of system analysis may not be sufficient to solve increasingly complex engineering problems.

\section{Methodology}

Methodical support for the design of technological processes (TP) should be based on the theory of technical systems. Therefore, it seems necessary to use the apparatus of this theory to formalize the basic concepts and principles of the technological processes design [1]. There are various definitions of the term "system". In this case, the aggregate of a finite set of interrelated elements, picked out from the environment and interacting with it, as a single whole, to solve a specific problem, is named a system.

The elements of a technological process system are actions or elements of the type "process" (operations, transitions). The relations connecting the elements of the type "process" in the system are temporary. There are serial, parallel and parallel-serial types of relations.

\subsection{Analysis of technological process of manufacturing the roller of brake rigging of the railway wagon}

Designing of models is considered on the simplest example of the technological process of manufacturing the roller of brake rigging of the railway wagon.

Let the technological process consists of one operation, which includes four transitions:

Transition No. 1 - installation of the workpiece on a lathe, Transition No. 2 - surface treatment,

Transition No. 3 - control,

Transition No. 4 - the removal of the part.

Consider the systematized logical model. This technological process can be represented as follows:

$T P 1=\{$ Ind, Prp, Atr, Inp, Out, Str $\}$,

where:

Ind - system designation and name,

Prp - targets of system,

Atr - attributes or system-wide characteristics,

Inp - system input,

Out - system output,

Str - system structure.

All the characteristics of the system for this case are here described in more details.

1. Ind

1.1 The name of the system is "Technological process of manufacturing the roller of brake rigging of wagon".

1.2 System Designation - «TP1». 
2. Prp - the purpose of the system is to manufacture the part in accordance with the requirements of the design documentation.

3. Atr - attributes or the system-wide characteristics are the signs by which systems can be classified and identified. These signs include the type of the details for manufacturing - the roller, the type of the processing - processing by cutting and the complexity of the technological process - simple.

4. Inp - the input of the technological process comes billet of detail with certain properties, as well as some production resources.

5. Out - the output of the technological process is the manufactured detail, as well as the production waste.

6. $\quad S t r=\langle E, R>$, where:

$\mathrm{E}$ - set of elements of the system,

$\mathrm{R}$ - set of relations connecting elements of the system.

A systematic process model can be represented graphically using a schematic (see Figure 1).

In Figure 1 are marked:

$P_{W}=\left\{P_{W}^{1}, P_{W}^{2}, \ldots, P_{W}^{n}\right\}$ - parameters of the workpiece, $P_{P}=\left\{P_{P}^{1}, P_{P}^{2}, \ldots, P_{P}^{n}\right\}$ - parameters of the product,

$R$ - production resources.

Production resources can be represented by the following four characteristics:

$R=<D, T, L, M>$,

where:

$D=\{D 1, D 2, \ldots, D n\}-$ technological equipment,

$T=\{T 1, T 2, \ldots, T n\}-$ technological rigging,

$L=\{L 1, L 2, \ldots, L n\}$ - labor resources,

$M=\{M 1, M 2, \ldots, M n\}-$ materials and components.

The state of the object in the manufacturing process is determined using operations of control. Those operations change the amount of information about manufactured object state without changing the physical state or its location.

\subsection{Creating a conceptual model}

The next step in modeling the technological process is to create its conceptual model [2], which determines the structure of the system. The structure of the system can be represented as a hierarchy diagram (see Figure 2):

$S t r=<E, R>$,

where:

$E=\left\{e_{1}, e_{2}, e_{3}, e_{4}\right\}$ - the transitions of the technological process which are represented as the set of vertices of the hierarchy diagram of system structure (Str), $e_{1}$ - installation of the workpiece on a lathe,

$e_{2}$-surface treatment,

$e_{3}$ - control,

$e_{4}$ - part removing,

$R\left\{r_{1}, r_{2}, r_{3}\right\}$ - the set of edges of the hierarchy diagram of the system structure Str, connecting in time the transitions of the technological process, $r_{1}=\left(e_{1}, e_{2}\right)$; $r_{1}=\left(e_{2}, e_{3}\right) ; r_{1}=\left(e_{3}, e_{4}\right)$.

This model can be represented by a set of related tables with parameters of elements of the technological process. The parameters for each element (transition) must uniquely identify it. The relationship of the transition parameter tables is determined by the time sequence of their execution [3].

In the technological process of manufacturing the roller of brake rigging of the railway wagon transitions are made sequentially and the parameters of the transitions are shown in Table 1.

\subsection{The construction of mathematical models}

The construction of mathematical models is considered next. To simulate this technological process, the two types of mathematical models are used: structural and functional.

The structural mathematical model can be represented as a hierarchy diagram (Figure 3).

$T=\langle S, E>$

where:

$E=\left\{e_{1}, e_{2}, e_{3}, e_{4}\right\}-$ are denoted transitions in the form of a set of $\operatorname{arcs}$,

$S=\left\{s_{1}, s_{2}, s_{3}, s_{4}, s_{5}\right\}$ - vertex set marking the state of the part,

for this technological process:

$s_{1}=P_{W}$ - parameters characterizing the initial state of the part,

$s_{2}$ - parameters characterizing the state of the part after the first transition (the workpiece is installed on the machine),

$s_{3}=s_{4}$ - parameters characterizing the state of the part after the second and third transition, since the control could not change the state of the part,

$s_{5}=P_{P}$ - parameters characterizing the state of the finished product.

The final step in creating a model of the technological process is the compilation of functional mathematical models of its elements. The task of modeling at this level is a quantitative analysis of the properties of objects, that is, the definition of dependencies of some parameters on others.

In particular, for the transition number 2 "Surface Treatment", one can create a mathematical functional model expressing the dependence of the basic time on 
Table 1 The parameters of the technological process of manufacturing the roller of brake rigging of the railway wagon transitions

\begin{tabular}{|c|c|}
\hline \multicolumn{2}{|c|}{ Transition No. 1 - installation of the workpiece on a lathe (vertex $e_{1}$ ) } \\
\hline $\mathrm{N}$ & number of transition \\
\hline $\mathrm{T} 1$ & device code \\
\hline $\mathrm{T} 2$ & code of assembly and locksmith's tool \\
\hline $\mathrm{O}$ & transition content \\
\hline $\mathrm{T}_{\text {time }}$ & transition time \\
\hline \multicolumn{2}{|c|}{ Transition No. 2 - surface treatment (vertex $\mathrm{e}_{2}$ ) } \\
\hline $\mathrm{N}$ & number of transition \\
\hline M & material grade code \\
\hline $\mathrm{T}$ & tool code \\
\hline $\mathrm{D}$ & detail diameter \\
\hline $\mathrm{L}$ & length of working stroke \\
\hline $\mathrm{t}$ & cutting depth \\
\hline $\mathrm{i}$ & number of passes \\
\hline $\mathrm{S}$ & cutting tool feed \\
\hline $\mathrm{n}$ & rotation frequency of a spindle \\
\hline $\mathrm{v}$ & cutting speed \\
\hline $\mathrm{t}_{\mathrm{a}}$ & auxiliary transition time \\
\hline$t_{m}$ & the main transition time \\
\hline \multicolumn{2}{|c|}{ Transition No. 3 - control (vertex $\mathrm{e}_{3}$ ) } \\
\hline $\mathrm{N}$ & number of transition \\
\hline $\mathrm{T}$ & measuring instrument code \\
\hline $\mathrm{O}$ & transition content \\
\hline \multicolumn{2}{|c|}{ Transition No. 4 - the removal of the part (vertex e e $_{4}$} \\
\hline $\mathrm{N}$ & number of transition \\
\hline $\mathrm{T}$ & code of assembly and locksmith's tool \\
\hline $\mathrm{O}$ & transition content \\
\hline
\end{tabular}

the transition from the length of the working stroke, the spindle rotationalspeed and feed:

$t_{O}=\frac{L}{n \cdot S}$

where:

$t_{0}$ - main transition time, minutes,

$L$ - length of the working stroke, mm,

$n$ - rotation frequency of the spindle, rotation per minute,

$S$ - cutting tool feed, mm per rotation.

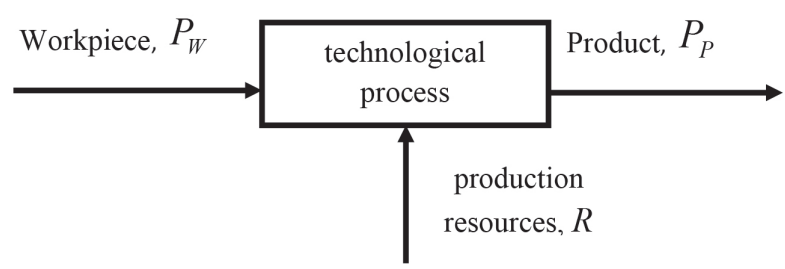

Figure 1 Process flow scheme

\section{Conclusions}

It should be noted that application of the proposed methods for constructing models of technological processes of production, repair and manufacture of objects in machine buildings, for developing the mathematical models of CAD systems, allows estimating the time costs at each production stage, as well as optimizing the process by combining operations and transitions at the design stage.

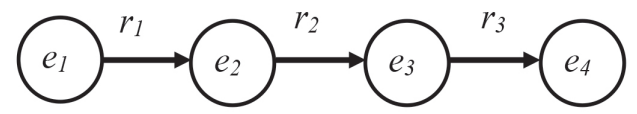

Figure 2 Technological process structure

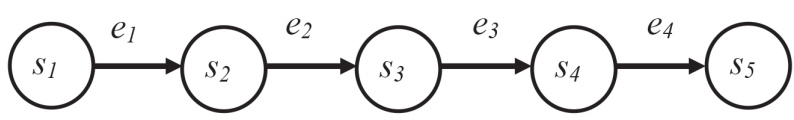

Figure 3 Structural mathematical model of the technological process 


\section{Acknowledgement}

The above methodology is used at the "non-traction rolling stock" department of Russian University of
Transport (RUT MIIT) when developing elements of the computer-aided design system for technological processes of production, repair and technical maintenance of objects machine buildings, such as the railway rolling stock.

\section{References}

[1] SERGEEV, K. A. Informatization of technological preparation of wagon-repair production (in Russian). Zheleznodorozhnyi transport. 2004, 10, p. 74. ISSN 0044-4448.

[2] SERGEEV, K. A. Modern approach to the formation of models of technological processes of wagon repairs (in Russian). Bulletin of the All-Russian Research Institute of Railway Transport. 2005, 1, p. 3. ISSN 2223-9731.

[3] SERGEEV, K. A., GUNDAEV, I. V., SIDOROV, E. S. Information and software technologist repair company (in Russian). Science and Technology of Transport. 2011, 2, p. 97-101. ISSN 2074-9325. 